\title{
含非完好界面的非线性弹性波超材料中SH波的 非互易传输
}

李珍妮 ${ }^{1}$, 王毅泽 ${ }^{*}$, 汪越胜 ${ }^{1,2}$

1. 北京交通大学土木建筑工程学院力学系, 北京 100044;

2. 天津大学机械工程学院力学系, 天津 300350

*联系人, E-mail: wangyize@tju.edu.cn

2019-09-03 收稿, 2019-10-31 修回, 2019-10-31 接受, 2019-12-30 网络版发表

国家自然科学基金(11532001)、中德科学中心中德合作研究项目(GZ 1355)和德国研究基金(ZH 15/27-1)资助

摘要 研究了含非完好界面的非线性弹性波超材料中的非互易传输现象. 通过结构的非对称性和材料的非线性打 破了经典弹性波系统中的互易性原理. 入射的反平面剪切(shear horizontal, SH)波与材料的非线性效应相互作用产 生二次谐波. 根据Bloch定理, 利用刚度矩阵法得到了基频波和二次谐波的带隙和透射系数。通过与完好界面情况 下的结果对比, 讨论了界面特性对层状周期结构中 SH波的带隙和非互易传输的影响. 本研究有助于设计具有非互 易传输性能的弹性波超材料器件.

关键词非线性弹性波超材料, 非互易传输, 二次谐波, 非完好界面, 带隙

近些年来，声子晶体和弹性波超材料作为一种人 工周期结构受到了国内外研究者的广泛关注 ${ }^{[1 \sim 3]}$. 这些 结构可以实现波的调控，并且具有传统材料所不具备 的特性, 如带隙 ${ }^{[4]}$ 、弹性波斗篷 ${ }^{[5]}$ 和负折射 ${ }^{[6]}$ 等. 在实 际应用中, 上述波动现象有助于设计先进器件. 其中一 个众所周知的特性为弹性波带隙, 即在特定的频率范 围内弹性波无法进行传播. 基于这一特性, 能够实现许 多工程应用, 例如滤波器以及声振隔离等.

材料非线性的存在，使得非线性波将带来新的现 象和有趣的传播行为, 因此受到了越来越多研究者的 广泛关注 ${ }^{[7 ~ 9]}$. 我们知道, 非线性现象一个重要的特性 是高阶谐波的产生. Deng和Xiang ${ }^{[10]}$ 研究了层状结构中 基于SH波与材料非线性相互作用产生的非线性 Lamb 波的传输特性. Liang等人 ${ }^{[11]}$ 结合声子晶体的带隙特性 和声学材料非线性实现了声波二极管. 弹性波二极管
或非互易传输是一种新的现象, 即弹性波在一个方向 可以传播, 但是在另一个方向则被截止 ${ }^{[12 \sim 14]}$.

周期结构的研究通常是建立在完好界面的基础上, 即位移和应力分量在界面处是连续的. 以往的研究工 作中，讨论了人射SH波在含完好界面的非线性弹性波 超材料中的非互易传输 ${ }^{[15]}$. 然而在实际应用中, 层状结 构中相邻层间的界面可呈现非完好特征. 理论研究结 果表明，非完好界面对结构的带隙和频散关系的影响 显著 ${ }^{[16,17]}$. 由于弹性波的非互易传输现象依赖于结构 的带隙, 因此研究非完好界面对非互易传输的影响将 显得十分必要. 本文结合声子晶体的带隙特性与材料 的非线性实现了含非完好界面结构中SH波的非互易传 输, 讨论了非完好界面对弹性波传输特性的影响, 实现 了其对非线性声子晶体和弹性波超材料中带隙和非互 易传输的调控. 


\section{1 模型和波动方程}

图1(a)给出了由两种不同的非线性材料A和B周期 排列而组成的非线性声子晶体. 该结构由 $m$ 个单胞构 成, 每个单胞的厚度为 $d=d_{1}+d_{2}$, 其中 $d_{1}$ 和 $d_{2}$ 表示子胞的 厚度. 局部坐标系如图1(a)所示, 其中 $\widehat{\boldsymbol{x}}, \widehat{\boldsymbol{y}}$ 和 $\widehat{\boldsymbol{z}}$ 为坐标系 的单位矢量. 结构中两个相邻固体层黏结而形成界面, 本文假设界面为非完好界面，即材料黏结面两侧物理 量不连续 ${ }^{[17]}$. 将非完好界面等效为力学弹簧模型(图1 (b)), 即在界面处应力连续而位移不连续. 界面性质由 法向和切向界面刚度系数 $K_{\mathrm{N}}$ 和 $K_{\mathrm{T}}$ 表示.

非线性弹性波超材料的理论模型如图1(c)所示, 该 结构由两种线性材料周期交替而成的声子晶体和非线 性介质A组成. 通过引人非线性机理打破了整体结构的 对称性. 利用声子晶体的带隙特性和材料的非线性实 现了弹性波的非互易传输，即弹性波沿正向可以传播， 但是反向将被截止, 如图1(d)所示.

考虑以 $x$ 轴为极化方向的人射SH波沿 $y o z$ 平面内传 播. 由于存在材料的非线性，根据二阶微扰近似，可得 基频波和二次谐波的控制方程 ${ }^{[10]}$ 为

$\rho_{j} \frac{\mathrm{d} \boldsymbol{u}_{j}^{(1)}}{\mathrm{d} t^{2}}-\left(\lambda_{j}+2 \mu_{j}\right) \nabla\left(\nabla \cdot \boldsymbol{u}_{j}^{(1)}\right)$

$+\mu_{j} \nabla \times\left(\nabla \times \boldsymbol{u}_{j}^{(1)}\right)=0$,

$$
\begin{aligned}
& \rho_{j} \frac{\mathrm{d} \boldsymbol{u}_{j}^{(2)}}{\mathrm{d} t^{2}}-\left(\lambda_{j}+2 \mu_{j}\right) \nabla\left(\nabla \cdot \boldsymbol{u}_{j}^{(2)}\right) \\
& +\mu_{j} \nabla \times\left(\nabla \times \boldsymbol{u}_{j}^{(2)}\right)=\boldsymbol{F}\left(\boldsymbol{u}_{j}^{(1)}\right),
\end{aligned}
$$

其中, $\boldsymbol{u}_{j}^{(1)}$ 和 $\boldsymbol{u}_{j}^{(2)}$ 为基频波和二次谐波的矢量位移; 下标 $j$ 表示周期层状结构的子胞; $\rho_{j}$ 为材料的密度; $\lambda_{j}$ 和 $\mu_{j}$ 为二 阶弹性常数; $\nabla=\left(\partial / \partial x_{j}, \partial / \partial y_{j}, \partial / \partial z_{j}\right)$ 为Nabla算子; $t$ 为时 间; $\boldsymbol{F}\left(\boldsymbol{u}_{j}^{(1)}\right)$ 为二次谐波彻体力，由基频波和材料的非线 性相互作用而产生 ${ }^{[10,16]}$.

通过求解方程(1)得到基频波的位移解为

$$
\begin{aligned}
\boldsymbol{u}_{j}^{(1)}= & \widehat{\boldsymbol{x}}\left\{u_{j \mathrm{~T}_{1}}^{(1)} \exp \left[\mathrm{i} k^{(1)}\left(\alpha_{j \mathrm{~T}} y+z\right)-\mathrm{i} \omega t\right]\right. \\
& \left.+u_{j \mathrm{~T}_{2}}^{(1)} \exp \left[\mathrm{i} k^{(1)}\left(-\alpha_{j \mathrm{~T}} y+z\right)-\mathrm{i} \omega t\right]\right\},
\end{aligned}
$$

其中, $u_{j \mathrm{~T}_{1}}^{(1)}$ 和 $u_{j \mathrm{~T}}^{(1)}$ 为基频波的振幅; $\mathrm{i}=\sqrt{-1} ; k^{(1)}=\omega / c^{(1)}$; $\omega$ 和 $c^{(1)}$ 分别为频率和人射波的相速度; $\alpha_{j \mathrm{~T}}=\sqrt{c^{(1)} / c_{j \mathrm{~T}}-1} ; c_{j \mathrm{~T}}$ 为横波的波速. 后续计算中, 为简 化推导将省略公共项 $\exp (-\mathrm{i} \omega t)$.

初始SH波在结构中传播，与材料非线性相互作用 可产生二次谐波彻体力. 将基频位移表达式(3)代人方 程式(2)中, 可得二次谐波彻体力 ${ }^{[10]}$ 为

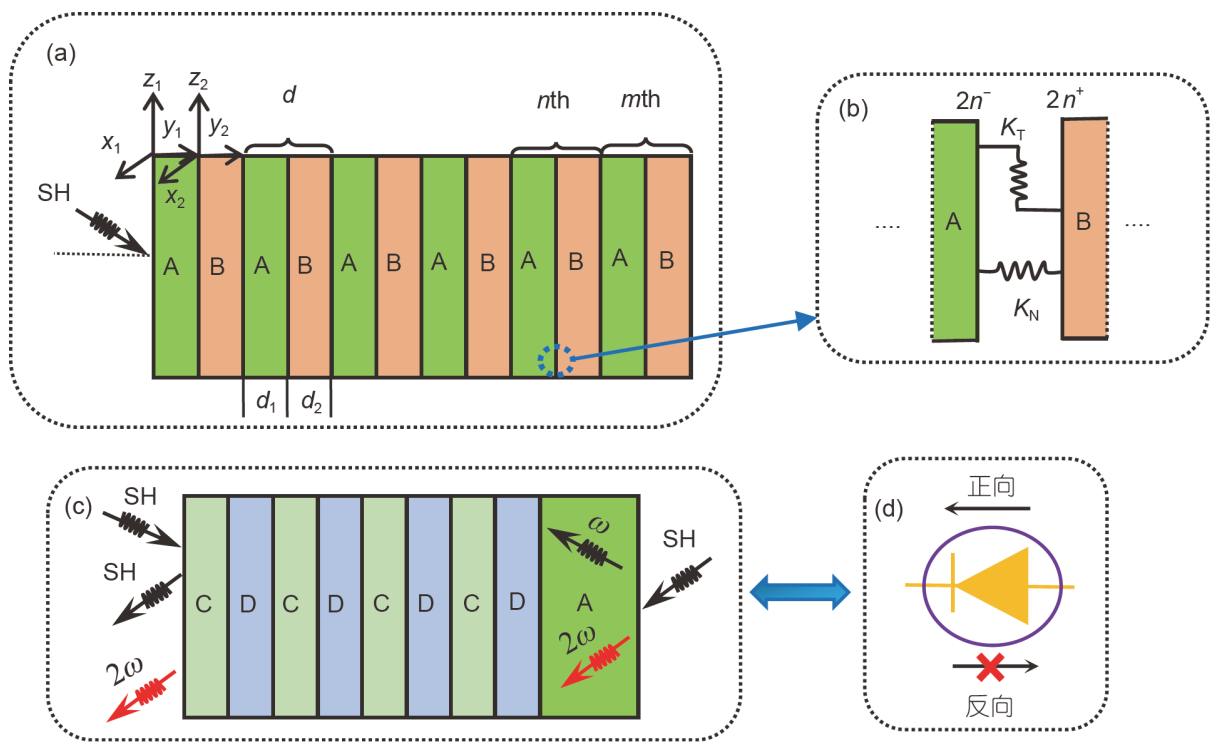

图 1 (网络版彩色)SH波人射时, 含非完好界面的非线性声子晶体和弹性波超材料的力学模型. (a) 非线性声子晶体及局部坐标系; (b) 非完好 界面模型; (c) 非线性弹性波超材料; (d) 非互易传输特性

Figure 1 (Color online) The nonlinear phononic crystal and elastic wave metamaterial with imperfect interfaces for an incident SH wave. (a) The nonlinear phononic crystal and the local coordinates; (b) the imperfect interfacial model; (c) the nonlinear elastic wave metamaterial; (d) its nonreciprocal transmission 


$$
\begin{aligned}
\boldsymbol{F}\left(\boldsymbol{u}_{j}^{(1)}\right)= & -\mathrm{i}\left(\lambda_{j}+2 \mu_{j}+\frac{A_{j}}{2}+B_{j}\right) K_{j \mathrm{~T}}^{3}\left(u_{j \mathrm{~T}_{1}}^{(1)}\right)^{2} \widehat{\boldsymbol{K}}_{j \mathrm{~T}_{1}}^{(1)} \exp \left[2 \mathrm{i} k^{(1)}\left(\alpha_{j \mathrm{~T}} y+z\right)\right] \\
& -\mathrm{i}\left(\lambda_{j}+2 \mu_{j}+\frac{A_{j}}{2}+B_{j}\right) K_{j \mathrm{~T}}^{3}\left(u_{j \mathrm{~T}_{2}}^{(1)}\right)^{2} \widehat{\boldsymbol{K}}_{j \mathrm{~T}_{2}}^{(1)} \exp \left[2 \mathrm{i} k^{(1)}\left(-\alpha_{j \mathrm{~T}} y+z\right)\right] \\
& -2 \mathrm{i} \bar{z}\left\{\mu_{j}+\frac{A_{j}}{4}-\left[1-2\left(\frac{c_{j \mathrm{~T}}}{c^{(1)}}\right)^{2}\right]\left(\lambda_{j}+\mu_{j}+\frac{A_{j}}{4}+B_{j}\right)\right\} u_{j \mathrm{~T}_{1}}^{(1)} u_{j \mathrm{~T}_{2}}^{(1)} k^{(1)} K_{j \mathrm{~T}}^{2} \exp \left(2 \mathrm{i} k^{(1)} z\right),
\end{aligned}
$$

其中, $\widehat{\boldsymbol{K}}_{j \mathrm{~T}_{1}}^{(1)}=k^{(1)}\left(\alpha_{j \mathrm{~T}} \widehat{\boldsymbol{y}}+\widehat{\boldsymbol{z}}\right)$ 和 $\widehat{\boldsymbol{K}}_{j \mathrm{~T}_{2}}^{(1)}=k^{(1)}\left(-\alpha_{j \mathrm{~T}} \widehat{\boldsymbol{y}}+\widehat{\boldsymbol{z}}\right)$ 为基频 位移分量的波矢量; $K_{j \mathrm{~T}}=\left|\widehat{\boldsymbol{K}}_{j \mathrm{~T}_{1}}^{(1)}\right|=\left|\widehat{\boldsymbol{K}}_{j \mathrm{~T}_{2}}^{(1)}\right| ; A_{j}$ 和 $B_{j}$ 为三阶弹 性常数.

结合式(4)可以得到:

$\widehat{\boldsymbol{x}} \cdot \boldsymbol{F}\left(\boldsymbol{u}_{j}^{(1)}\right)=0$.

由此可以推断出, 作为二次谐波的驱动源, 将会产 生一系列平面内二次谐波沿 $y o z$ 平面进行传播. 结合控 制方程式(2)可得二次谐波位移解为

$$
\begin{aligned}
\boldsymbol{u}_{j \mathrm{~T}}^{(2)}= & \left(\widehat{\boldsymbol{K}}_{j \mathrm{~T}_{1}}^{(2)} \times \widehat{\boldsymbol{x}}\right) u_{j \mathrm{~T}_{1}}^{(2)} \exp \left[\mathrm{i} k^{(2)}\left(\beta_{j \mathrm{~T}} y+z\right)\right] \\
& +\left(\widehat{\boldsymbol{K}}_{j \mathrm{~T}_{2}}^{(2)} \times \widehat{\boldsymbol{x}}\right) u_{j \mathrm{~T}_{2}}^{(2)} \exp \left[\mathrm{i} k^{(2)}\left(-\beta_{j \mathrm{~T}} y+z\right)\right] \\
& +\widehat{\boldsymbol{K}}_{j \mathrm{~L}_{1}}^{(2)} u_{j \mathrm{~L}}^{(2)} \exp \left[\mathrm{i} k^{(2)}\left(\beta_{j \mathrm{~L}} y+z\right)\right] \\
& +\widehat{\boldsymbol{K}}_{j \mathrm{~L}_{2}}^{(2)} u_{j \mathrm{~L}_{2}}^{(2)} \exp \left[\mathrm{i} k^{(2)}\left(-\beta_{j \mathrm{~L}} y+z\right)\right],
\end{aligned}
$$

其中, $\widehat{\boldsymbol{K}}_{j Q p}^{(2)}(Q=\mathrm{T}, \mathrm{L} ; p=1,2)$ 为二次谐波位移分量的波矢 量; $u_{j Q p}^{(2)}$ 为待解的振幅; $\beta_{j Q}=\sqrt{c^{(2)} / c_{j Q}-1} ; c^{(2)}$ 为二次谐 波人射波的相速度; $c_{j \mathrm{~L}}$ 为纵波的波速; $k^{(2)}$ 为二次谐波的 波数.

\section{2 含非完好界面的刚度矩阵}

根据本构关系, 可以写出剪切应力表达式为

$$
\tau_{j x y}^{(1)}=\mu_{j} \frac{\partial u_{j}^{(1)}}{\partial y} .
$$

将基频波位移解(式(3))代人上式得

$$
\begin{aligned}
\tau_{j x y}^{(1)}= & \mathrm{i} \mu_{j} k^{(1)} \alpha_{j \mathrm{~T}} u_{j \mathrm{~T}_{1}}^{(1)} \exp \left[\mathrm{i} k^{(1)}\left(\alpha_{j \mathrm{~T}} y+z\right)\right] \\
& -\mathrm{i} \mu_{j} k^{(1)} \alpha_{j \mathrm{~T}} u_{j \mathrm{~T}_{2}}^{(1)} \exp \left[\mathrm{i} k^{(1)}\left(-\alpha_{j \mathrm{~T}} y+z\right)\right] .
\end{aligned}
$$

完好界面是指力学参数在界面处均连续的界面. 如果部分或全部力学参数在界面处不连续则称之为非 完好界面. 在具体计算中, 可将非完好界面假设为一种 无厚度的薄层 ${ }^{[17]}$. 本文将非完好界面等效为力学弹簧
模型, 即应力分量在边界处连续而位移分量不连续. 因 此, 基频波在界面 $2 n$ 和 $2 n+1$ 处所满足的边界条件为

$\left.\tau_{j x y}^{(1)}\right|_{y=2 n^{+} / 2 n+1^{+}}$

$=\left.\tau_{j x y}^{(1)}\right|_{y=2 n^{-} / 2 n+1^{-}}$

$=\left.\tau_{j x y}^{(1)}\right|_{y=2 n / 2 n+1}$,

$\left.\tau_{j x y}^{(1)}\right|_{y=2 n / 2 n+1}$

$=K_{\mathrm{T}}\left(\left.u_{j}^{(1)}\right|_{y=2 n^{+} / 2 n+1^{+}}-\left.u_{j}^{(1)}\right|_{y=2 n^{-} / 2 n+1^{-}}\right)$.

根据本构关系, 子胞左右边界的状态向量可由刚 度矩阵表示为如下形式:

$$
\begin{aligned}
& \left\{\begin{array}{c}
\left.\tau_{j x y}^{(1)}\right|_{y=2 n-1} \\
\left.\tau_{j x y}^{(1)}\right|_{y=2 n^{-}}
\end{array}\right\}=\boldsymbol{K}^{\mathrm{A}}\left\{\begin{array}{c}
\left.u_{j}^{(1)}\right|_{y=2 n-1} \\
\left.u_{j}^{(1)}\right|_{y=2 n^{-}}
\end{array}\right\}, \\
& \left\{\begin{array}{c}
\left.\tau_{j x y}^{(1)}\right|_{y=2 n^{+}} \\
\left.\tau_{j x y}^{(1)}\right|_{y=2 n+1}
\end{array}\right\}=\boldsymbol{K}^{\mathrm{B}}\left\{\begin{array}{c}
\left.u_{j}^{(1)}\right|_{y=2 n^{+}} \\
\left.u_{j}^{(1)}\right|_{y=2 n+1}
\end{array}\right\},
\end{aligned}
$$

其中, $\boldsymbol{K}^{W}(W=\mathrm{A}, \mathrm{B})$ 为子胞的刚度矩阵, 其分量为

$$
\begin{aligned}
& K_{11}^{W}=\frac{-\mathrm{i} \mu_{j} \omega \alpha_{j \mathrm{~T}}\left(r d_{j 1}+r d_{j 2}\right)}{c^{(1)}\left(r d_{j 1}-r d_{j 2}\right)}, \\
& K_{12}^{W}=\frac{2 \mathrm{i} \mu_{j} \omega \alpha_{j \mathrm{~T}}}{c^{(1)}\left(r d_{j 1}-r d_{j 2}\right)}, \\
& K_{21}^{W}=\frac{-2 \mathrm{i} \mu_{j} \omega \alpha_{j \mathrm{~T}} r d_{j \mathrm{r}} r d_{j 2}}{c^{(1)}\left(r d_{j 1}-r d_{j 2}\right)}, \\
& K_{22}^{W}=\frac{\mathrm{i} \mu_{j} \omega \alpha_{j \mathrm{~T}}\left(r d_{j 1}+r d_{j 2}\right)}{c^{(1)}\left(r d_{j 1}-r d_{j 2}\right)}, \\
& r d_{j 1}=\exp \left(\mathrm{i} \omega \alpha_{j \mathrm{~T}} d_{j} / c^{(1)}\right), \\
& r d_{j 2}=\exp \left(-\mathrm{i} \omega \alpha_{j \mathrm{~T}} d_{j} / c^{(1)}\right) .
\end{aligned}
$$

将式(3)和(8)代人边界条件式(9), 可得刚度矩阵 $\boldsymbol{K}^{\mathrm{I}}$ 为

$$
\boldsymbol{K}^{\mathrm{I}}=\left[\begin{array}{ll}
-K_{\mathrm{T}} & K_{\mathrm{T}} \\
-K_{\mathrm{T}} & K_{\mathrm{T}}
\end{array}\right] \text {. }
$$

联立式(10), (11)和(12), 可得一个单胞的局部刚度矩 阵为 
$\boldsymbol{K}=\left[\begin{array}{ll}K_{11}^{\mathrm{AIB}}+K_{12}^{\mathrm{AIB}}\left(K_{11}^{\mathrm{I}}-K_{22}^{\mathrm{AIB}}\right)^{-1} K_{21}^{\mathrm{AIB}} & -K_{12}^{\mathrm{AIB}}\left(K_{11}^{\mathrm{I}}-K_{22}^{\mathrm{AIB}}\right)^{-1} K_{12}^{\mathrm{I}} \\ K_{21}^{\mathrm{I}}\left(K_{11}^{\mathrm{I}}-K_{22}^{\mathrm{AIB}}\right)^{-1} K_{21}^{\mathrm{AIB}} & K_{22}^{\mathrm{I}}-K_{21}^{\mathrm{I}}\left(K_{11}^{\mathrm{I}}-K_{22}^{\mathrm{AIB}}\right)^{-1} K_{12}^{\mathrm{I}}\end{array}\right]$,

其中,

$K_{11}^{\mathrm{AIB}}=K_{11}^{\mathrm{AI}}+K_{12}^{\mathrm{AI}}\left(K_{11}^{\mathrm{B}}-K_{22}^{\mathrm{AI}}\right)^{-1} K_{21}^{\mathrm{AI}}, \quad K_{12}^{\mathrm{AIB}}=-K_{12}^{\mathrm{AI}}\left(K_{11}^{\mathrm{B}}-K_{22}^{\mathrm{AI}}\right)^{-1} K_{12}^{\mathrm{B}}$,

$K_{21}^{\mathrm{AIB}}=K_{21}^{\mathrm{B}}\left(K_{11}^{\mathrm{B}}-K_{22}^{\mathrm{AI}}\right)^{-1} K_{21}^{\mathrm{AI}}, \quad K_{22}^{\mathrm{AIB}}=K_{22}^{\mathrm{B}}-K_{21}^{\mathrm{B}}\left(K_{11}^{\mathrm{B}}-K_{22}^{\mathrm{AI}}\right)^{-1} K_{12}^{\mathrm{AI}}$,

$K_{11}^{\mathrm{AI}}=K_{11}^{\mathrm{A}}+K_{12}^{\mathrm{A}}\left(K_{11}^{\mathrm{I}}-K_{22}^{\mathrm{A}}\right)^{-1} K_{21}^{\mathrm{A}}, \quad K_{12}^{\mathrm{AI}}=-K_{12}^{\mathrm{A}}\left(K_{11}^{\mathrm{I}}-K_{22}^{\mathrm{A}}\right)^{-1} K_{12}^{\mathrm{I}}$,

$K_{21}^{\mathrm{AI}}=K_{21}^{\mathrm{I}}\left(K_{11}^{\mathrm{I}}-K_{22}^{\mathrm{A}}\right)^{-1} K_{21}^{\mathrm{A}}$, $K_{22}^{\mathrm{AI}}=K_{22}^{\mathrm{I}}-K_{21}^{\mathrm{I}}\left(K_{11}^{\mathrm{I}}-K_{22}^{\mathrm{A}}\right)^{-1} K_{12}^{\mathrm{A}}$.

由于结构在 $y$ 轴方向上具有周期性，根据Bloch定 理, 可得应力和位移分量的关系如下:

$\left\{\begin{array}{c}u_{j}^{(1)} \\ \tau_{j x y}^{(1)}\end{array}\right\}_{2 n+1}=\mathrm{e}^{\mathrm{i} k d}\left\{\begin{array}{c}u_{j}^{(1)} \\ \tau_{j x y}^{(1)}\end{array}\right\}_{2 n-1}$.

通过矩阵运算可得传递矩阵 $\boldsymbol{T}$ 的表达式为

$\boldsymbol{T}=\left[\begin{array}{cc}-K_{12}^{-1} K_{11} & K_{12}^{-1} \\ K_{21}-K_{22} K_{12}^{-1} K_{11} & K_{22} K_{12}^{-1}\end{array}\right]$.
结合式(15)和(16)可得系统的特征方程:

$\left|\boldsymbol{T}-\mathrm{e}^{\mathrm{i} k d} \boldsymbol{I}\right|=0$.

通过求解特征值方程式(17), 可以得到系统的带隙 特征. 当存在实数解时, 则表示在该频率范围内有弹性 波能够进行传播, 即为通带, 反之则为禁带.

考虑具有 $m$ 个单胞的层状周期结构, 假设 $\boldsymbol{K}^{m-1}$ 为前 $m-1$ 个单胞的刚度矩阵, $\boldsymbol{K}^{M}$ 为第 $m$ 个单胞的刚度矩阵, 根据迭代法可以得到周期结构的整体刚度矩阵 $\boldsymbol{K}^{m}$ 为 ${ }^{[18]}$

$\boldsymbol{K}^{m}=\left[\begin{array}{ll}K_{11}^{m-1}+K_{12}^{m-1}\left(K_{11}^{M}-K_{22}^{m-1}\right)^{-1} K_{21}^{M} & -K_{12}^{m-1}\left(K_{11}^{M}-K_{22}^{m-1}\right)^{-1} K_{12}^{M} \\ K_{21}^{M}\left(K_{11}^{M}-K_{22}^{m-1}\right)^{-1} K_{21}^{m-1} & K_{22}^{M}+K_{12}^{M}\left(K_{11}^{M}-K_{22}^{m-1}\right)^{-1} K_{21}^{M}\end{array}\right]$.

对于人射 $\mathrm{SH}$ 波，在层状周期结构的人射边界处的 位移场由一个人射波和一个反射波构成，位移解可以 表示为

$$
\begin{aligned}
u_{(1)}= & A_{\mathrm{I}} \exp \left[\mathrm{i} k^{(1)}\left(\alpha_{(1) \mathrm{T}} y+z\right)\right] \\
& +A_{\mathrm{R}} \exp \left[\mathrm{i} k^{(1)}\left(-\alpha_{(1) \mathrm{T}} y+z\right)\right],
\end{aligned}
$$

其中, 下标(1)表示人射边界, $A_{\mathrm{I}}$ 和 $A_{\mathrm{R}}$ 分别为人射波和反 射波的振幅.

相应的透射边界处位移为

$u_{(2)}=A_{\mathrm{T}} \exp \left[\mathrm{i} k^{(1)}\left(\alpha_{(2) \mathrm{T}} y+z\right)\right]$,

其中, 下标(2)表示透射边界, $A_{\mathrm{T}}$ 为透射波的振幅.

可得含有应力和位移的状态向量的整体刚度矩 阵为
因此, 可以得到透射系数和反射系数:

$\left\{\begin{array}{l}F_{\text {DTT }} \\ F_{\text {RTT }}\end{array}\right\}=\boldsymbol{M}^{-1} \boldsymbol{N}$,

其中, $F_{\mathrm{DTT}}=A_{\mathrm{T}} / A_{\mathrm{I}}$ 和 $F_{\mathrm{RTT}}=A_{\mathrm{R}} / A_{\mathrm{I}}$ 分别为透射系数和反射 系数, 系数矩阵 $M$ 和 $N$ 中的元素为

$M_{11}=K_{12}^{m}$,

$M_{12}=K_{11}^{m}+\mathrm{i} k^{(1)} \alpha_{(1) \mathrm{T}} \mu_{(1)}$,

$M_{21}=K_{22}^{m}-\mathrm{i} k^{(1)} \alpha_{(2) \mathrm{T}} \mu_{(2)}$,

$M_{22}=K_{21}^{m}$,

$N_{11}=\mathrm{i} k^{(1)} \alpha_{(1) \mathrm{T}} \mu_{(1)}-K_{11}^{m}$,

$N_{21}=-K_{21}^{m}$.

基频波与材料非线性相互作用将产生二次谐波, 其位移表达式(6)可分解为 $y$ 轴和 $z$ 轴两个方向的分量: 


$$
\begin{aligned}
u_{j y}^{(2)}= & -\sin \theta_{j \mathrm{~T}} u_{j \mathrm{~T}_{1}}^{(2)} s d_{j 1}+\sin \theta_{j \mathrm{~T}} u_{j \mathrm{~T}_{2}}^{(2)} s d_{j 2} \\
& +\cos \theta_{j \mathrm{~L}} u_{j \mathrm{~L}_{1}}^{(2)} s d_{j 3}-\cos \theta_{j \mathrm{~L}} u_{j \mathrm{~L}_{2}}^{(2)} s d_{j 4}, \\
u_{j z}^{(2)}= & \cos \theta_{j \mathrm{~T}} u_{j \mathrm{~T}_{1}}^{(2)} s d_{j 1}+\cos \theta_{j \mathrm{~T}} u_{j \mathrm{~T}_{2}}^{(2)} s d_{j 2} \\
& +\sin \theta_{j \mathrm{~L}} u_{j \mathrm{~L}_{1}}^{(2)} s d_{j 3}+\sin \theta_{j \mathrm{~L}} u_{j \mathrm{~L}_{2}}^{(2)} s d_{j 4},
\end{aligned}
$$

其中,

$$
\begin{aligned}
s d_{j 1} & =\exp \left[\mathrm{i} k^{(2)}\left(\beta_{j \mathrm{~T}} y+z\right)\right], \\
s d_{j 2} & =\exp \left[\mathrm{i} k^{(2)}\left(-\beta_{j \mathrm{~T}} y+z\right)\right], \\
s d_{j 3} & =\exp \left[\mathrm{i} k^{(2)}\left(\beta_{j \mathrm{~L}} y+z\right)\right], \\
s d_{j 4} & =\exp \left[\mathrm{i} k^{(2)}\left(-\beta_{j \mathrm{~L}} y+z\right)\right], \\
\sin \theta_{j Q} & =c_{j Q} / c^{(2)}, \\
\cos \theta_{j Q} & =\beta_{j Q} \sin \theta_{j Q} .
\end{aligned}
$$

应力分量可以表示为

$\tau_{j z y}^{(2)}=\mu_{j}\left(\frac{\partial u_{j y}^{(2)}}{\partial z}+\frac{\partial u_{j z}^{(2)}}{\partial y}\right)$,

$\sigma_{j y y}^{(2)}=\left(\lambda_{j}+2 \mu_{j}\right) \frac{\partial u_{j y}^{(2)}}{\partial y}+\lambda_{j} \frac{\partial u_{j z}^{(2)}}{\partial z}$.

上述分量的详细表达式可参见文献[15]. 根据非完 好界面的力学性质, 二次谐波在边界处所满足的边界 条件为

$\left.\tau_{j z y}^{(2)}\right|_{y=2 n^{+} / 2 n+1^{+}}=\left.\tau_{j z y}^{(2)}\right|_{y=2 n^{-} / 2 n+1}=\left.\tau_{j z y}^{(2)}\right|_{y=2 n / 2 n+1}$,

$\left.\sigma_{j y y}^{(2)}\right|_{y=2 n^{+} / 2 n+1^{+}}=\left.\sigma_{j y y}^{(2)}\right|_{y=2 n^{-} / 2 n+1}=\left.\sigma_{j y y}^{(2)}\right|_{y=2 n / 2 n+1}$,

$\left.\tau_{j z y}^{(2)}\right|_{y=2 n / 2 n+1}=K_{\mathrm{T}}\left[\left.u_{j z}^{(2)}\right|_{y=2 n^{+} / 2 n+1^{+}}+\left.u_{j z}^{(2)}\right|_{y=2 n^{-} / 2 n+1^{-}}\right.$,

$\left.\sigma_{j y y}^{(2)}\right|_{y=2 n / 2 n+1}=K_{\mathrm{N}}\left[\left.\left.u_{j y}^{(2)}\right|_{y=2 n^{+} / 2 n+1^{+}} u_{j y}^{(2)}\right|_{y=2 n^{-} / 2 n+1^{-}}\right]$.

由式(24) (27), 可得二次谐波子胞和界面的刚度 矩阵为

$$
\left\{\begin{array}{l}
\left.\boldsymbol{\sigma}^{(2)}\right|_{y=2 n-1} \\
\left.\boldsymbol{\sigma}^{(2)}\right|_{y=2 n^{-}}
\end{array}\right\}=\boldsymbol{K}^{(2) A}\left\{\begin{array}{l}
\left.\boldsymbol{u}^{(2)}\right|_{y=2 n-1} \\
\left.\boldsymbol{u}^{(2)}\right|_{y=2 n^{-}}
\end{array}\right\},
$$$$
\left\{\begin{array}{l}
\left.\boldsymbol{\sigma}^{(2)}\right|_{y=2 n^{-}} \\
\left.\boldsymbol{\sigma}^{(2)}\right|_{y=2 n^{+}}
\end{array}\right\}=\boldsymbol{K}^{(2) I}\left\{\begin{array}{l}
\left.\boldsymbol{u}^{(2)}\right|_{y=2 n^{-}} \\
\left.\boldsymbol{u}^{(2)}\right|_{y=2 n^{+}}
\end{array}\right\},
$$$$
\left\{\begin{array}{c}
\left.\boldsymbol{\sigma}^{(2)}\right|_{y=2 n^{+}} \\
\left.\boldsymbol{\sigma}^{(2)}\right|_{y=2 n+1^{-}}
\end{array}\right\}=\boldsymbol{K}^{(2) B}\left\{\begin{array}{c}
\left.\boldsymbol{u}^{(2)}\right|_{y=2 n^{+}} \\
\left.\boldsymbol{u}^{(2)}\right|_{y=2 n+1^{-}}
\end{array}\right\},
$$$$
\left\{\begin{array}{l}
\left.\boldsymbol{\sigma}^{(2)}\right|_{y=2 n+1^{-}} \\
\left.\boldsymbol{\sigma}^{(2)}\right|_{y=2 n+1^{+}}
\end{array}\right\}=\boldsymbol{K}^{(2) I}\left\{\begin{array}{l}
\left.\boldsymbol{u}^{(2)}\right|_{y=2 n+1^{-}} \\
\left.\boldsymbol{u}^{(2)}\right|_{y=2 n+1^{+}}
\end{array}\right\},
$$

其中, $\boldsymbol{\sigma}^{(2)}=\left\{\begin{array}{ll}\tau_{j z y}^{(2)} & \sigma_{j y y}^{(2)}\end{array}\right\}^{\mathrm{T}}$ 和 $\boldsymbol{u}^{(2)}=\left\{\begin{array}{ll}u_{j y}^{(2)} & u_{j y}^{(2)}\end{array}\right\}^{\mathrm{T}}$ 分别为应 力和位移的状态向量; $\boldsymbol{K}^{(2) \mathrm{A}}$ 和 $\boldsymbol{K}^{(2) \mathrm{B}}$ 为子胞的刚度矩阵; $\boldsymbol{K}^{(2) I}$ 为界面的刚度矩阵, 可表示为

$$
\boldsymbol{K}^{(2) \mathrm{I}}=\left[\begin{array}{cccc}
-K_{\mathrm{N}} & 0 & K_{\mathrm{N}} & 0 \\
0 & -K_{\mathrm{T}} & 0 & K_{\mathrm{T}} \\
-K_{\mathrm{N}} & 0 & K_{\mathrm{N}} & 0 \\
0 & -K_{\mathrm{T}} & 0 & K_{\mathrm{T}}
\end{array}\right] .
$$

采用相似的推导过程，可得二次谐波作用时一 个单胞的局部刚度矩阵 $\boldsymbol{K}^{(2)}$ 以及 $m$ 个单胞的整体刚度 矩阵 $\boldsymbol{K}^{(2) m}$. 根据Bloch定理, 可得二次谐波情况的特 征方程，并可求解含非完好界面结构的非线性带隙 特性.

二次谐波横波模态在人射边界处由一个人射波和 两个反射波组成, 并可表示为

$$
\begin{aligned}
u_{(1) j y}= & -\sin \theta_{(1) \mathrm{T}} s d_{(1) 1}+\sin \theta_{(1) \mathrm{T}} s d_{(1) 2} H_{\mathrm{RTT}} \\
& -\cos \theta_{(1) \mathrm{L}} s d_{(1) 4} H_{\mathrm{RTL}}, \\
u_{(1) j \mathrm{z}}= & \cos \theta_{(1) \mathrm{T}} s d_{(1) 1}+\cos \theta_{(1) \mathrm{T}} s d_{(1) 2} H_{\mathrm{RTT}} \\
& +\sin \theta_{(1) \mathrm{L}} s d_{(1) 4} H_{\mathrm{RTL}},
\end{aligned}
$$

其中, $H_{\mathrm{RTT}}$ 和 $H_{\mathrm{RTL}}$ 分别为横波和纵波模态的反射系数.

透射边界处的位移为

$$
\begin{aligned}
u_{(2) j y}= & -\sin \theta_{(2) \mathrm{T}} s d_{(2) 1} H_{\mathrm{DTT}} \\
& +\cos \theta_{(2) \mathrm{L}} s d_{(2) 3} H_{\mathrm{DTL}}, \\
u_{(2) j z}= & \cos \theta_{(2) \mathrm{T}} s d_{(2) \mathrm{H}} H_{\mathrm{DTT}} \\
& +\sin \theta_{(2) \mathrm{L}} s d_{(2) 3} H_{\mathrm{DTL}},
\end{aligned}
$$

其中, $H_{\mathrm{DTT}}$ 和 $H_{\mathrm{DTL}}$ 分别为横波和纵波模态的透射系数.

由此可求得二次谐波情况时的反射和透射系数, 并计算出二次谐波的传输特性. 本文将人射波为二次 谐波纵波模态得到的透射系数定义为 $H_{\mathrm{DLT}}$ 和 $H_{\mathrm{DLL}}$.

\section{3 数值模拟和讨论}

图1(a)中的非线性声子晶体由两种非线性材料组 成，分别为 5052 铝合金 $(\mathrm{A})$ 和 304 不锈钢 $(\mathrm{B})^{[10,19]}$. 如 图1(c)所示, 非线性弹性波超材料由声子晶体和非线性 介质(A)组合而成，其中声子晶体由线性材料AZ31B镁 合金 $(C)$ 和TC4钛合金 $(D)$ 组成 ${ }^{[20]}$. 本文采用切向和法向 的界面刚度系数分别为 $K_{\mathrm{T}}=0.638 \times 10^{14} \mathrm{~N} / \mathrm{m}^{3}$ 和 $K_{\mathrm{N}}=$ $3.175 \times 10^{14} \mathrm{~N} / \mathrm{m}^{3[16,17]}$, 人射波的相速度 $c^{(1)}=c^{(2)}=$ 

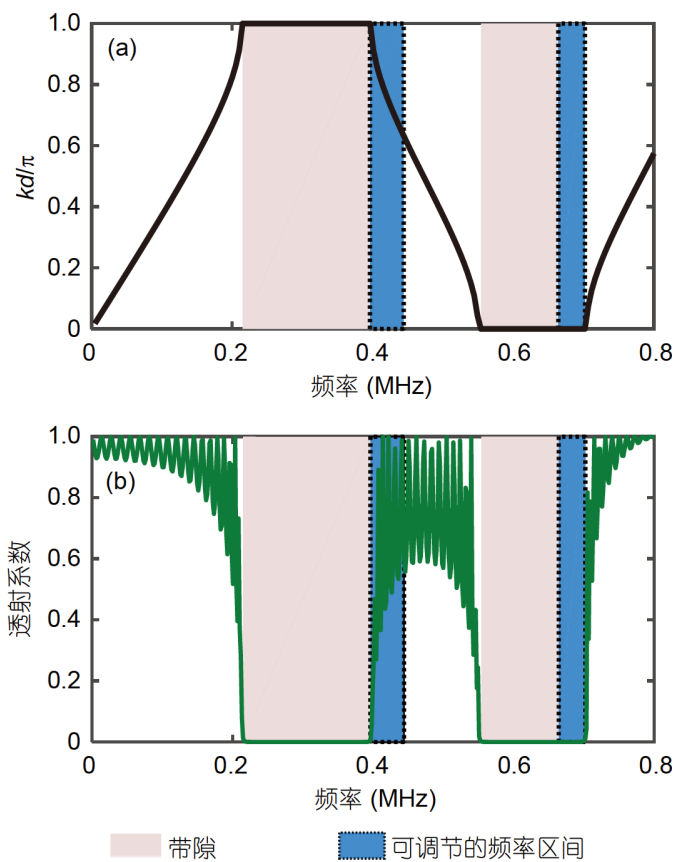

图 2 (网络版彩色)含非完好界面的非线性声子晶体中基频波的带 隙(a)和相应的透射系数(b)

Figure 2 (Color online) Band gap (a) and the transmission coefficient (b) of the fundamental wave in the nonlinear phononic crystal with imperfect interfaces

$5000 \mathrm{~m} / \mathrm{s}$, 厚度比 $d_{1} / d_{2}=1 / 1$. 对于周期层状结构, 假设其 包含 $m=20$ 个单胞.

图2给出了含非完好界面的非线性声子晶体中基 频波的带隙和透射系数, 图中深色阴影区域表示其与 完好界面结构带隙的对比结果. 从图2(a)可以看出，基 频波的带隙出现在频率区间 $0.22 \sim 0.40$ 和 $0.55 \sim$ $0.70 \mathrm{MHz}$ 内. 与完好界面情况相比较, 在非完好界面条 件下带隙的中心频率明显向低频区域移动，并且第二 阶带隙的宽度减小. 图2(b)表示相应的透射系数，当实 数解为零时对应于禁带, 反之则为通带. 结果表明, 带 隙的位置和宽度与图2(a)中的结果完全吻合.

图3给出了含非完好界面的非线性声子晶体中二 次谐波的带隙和透射系数. 可以看出, 图3(b)和(c)中透 射系数为零的频率区间与图3(a)中带隙的位置吻合较 好. 此外, 二次谐波带隙的变化与基频波的情况一致, 并且第二阶带隙宽度的变化更加明显. 完好界面时的 禁带由于界面特性的影响转变为通带. 因此, 非完好界 面可以作为一种调节周期层状结构中带隙位置和宽度 的有效方法.

图4给出了含非完好界面的非线性弹性波超材料 中基频波和二次谐波的带隙. 如图所示，频率区间
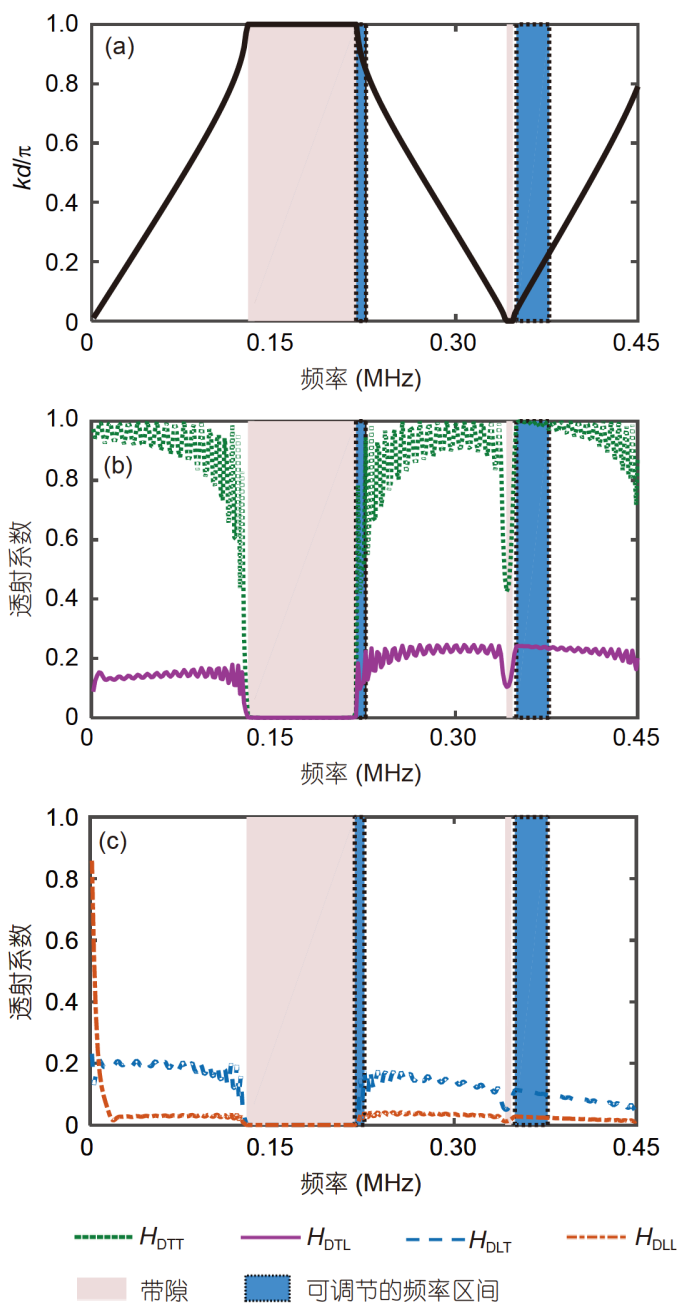

图 3 (网络版彩色)含非完好界面的非线性声子晶体中二次谐波的 带隙和相应的透射系数. (a) 带隙; (b) 二次横波所产生的横波和纵波; (c) 二次纵波所产生的横波和纵波的透射系数

Figure 3 (Color online) Band gap and transmission coefficients of the second harmonic in the nonlinear phononic crystal with imperfect interfaces. (a) The band gap; the transmission coefficients of the generated transverse and longitudinal waves for the incident transverse wave (b) and the incident longitudinal wave (c)
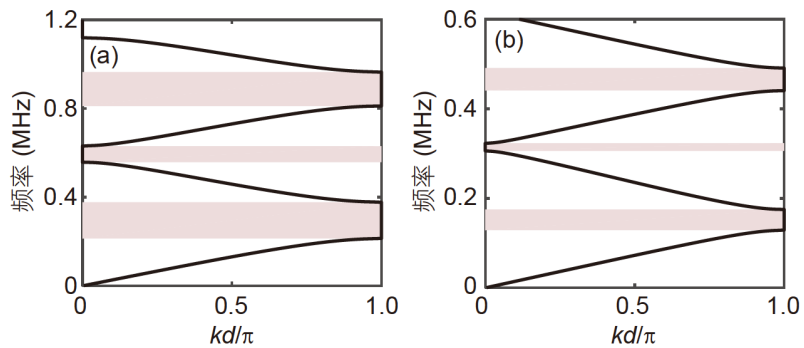

图 4 (网络版彩色)含非完好界面的非线性弹性波超材料中基频波 (a) 和二次谐波(b)的带隙

Figure 4 (Color online) Band gaps of the fundamental wave (a) and the second harmonic (b) in the nonlinear elastic wave metamaterial with imperfect interfaces 
$0.21 \sim 0.38,0.56 \sim 0.63$ 和 $0.81 \sim 0.96 \mathrm{MHz}$ 为基频波的禁带; 而0.13 0.18, 0.31 0.32和0.44 0.49 MHz则属于二次谐 波的禁带. 当基频波处于禁带而二次谐波处于通带时, 弹性波的非互易传输现象可以实现. 因此, 频率区间 $0.21 \sim 0.31$ 和 $0.32 \sim 0.38 \mathrm{MHz}$ 可视为非完好界面条件下 的非互易传输区间. 作为对比算例, 计算出含完好界面 周期结构的非互易区间为 $0.23 \sim 0.31$ 和 $0.34 \sim 0.40 \mathrm{MHz}$. 可以明显看出，界面特性使得非互易传输区间的中心 频率向低频区域移动.

当人射 $\mathrm{SH}$ 波从结构右边界人射时，与材料的非线 性相互作用将产生二次谐波. 此时系统中存在两种类 型的弹性波从右向左传播. 如图5所示，在非互易区间 0.21 0.31和0.32 0.38 MHz内，基频波的透射系数为零 而二次谐波的透射系数不为零. 因此, 二次谐波正向可 以进行传播. 当人射 $\mathrm{SH}$ 波从结构左边界人射时, 由于线 性材料不会产生非线性弹性波，二次谐波的透射系数 在整个频率区间内为零，使得反向没有弹性波可以通 过, 实现了非互易传输现象, 即弹性波在一个方向可以 传播而另一个方向则被截止.

\section{4 总结}

本文研究了含非完好界面的非线性声子晶体和弹 性波超材料的传输行为. 考虑 SH波人射情况且在结构 中传播时与材料非线性相互作用产生二次谐波，同时 结合带隙特性实现了弹性波的非互易传输. 进一步讨 论了非完好界面对带隙和非互易行为的影响. 研究结 果表明, 与完好界面情况相比, 含有非完好界面的结构 中带隙和非互易传输的中心频率明显向低频区域移动. 本文研究有助于设计具有可调节功能的弹性波非互易 传输器件.
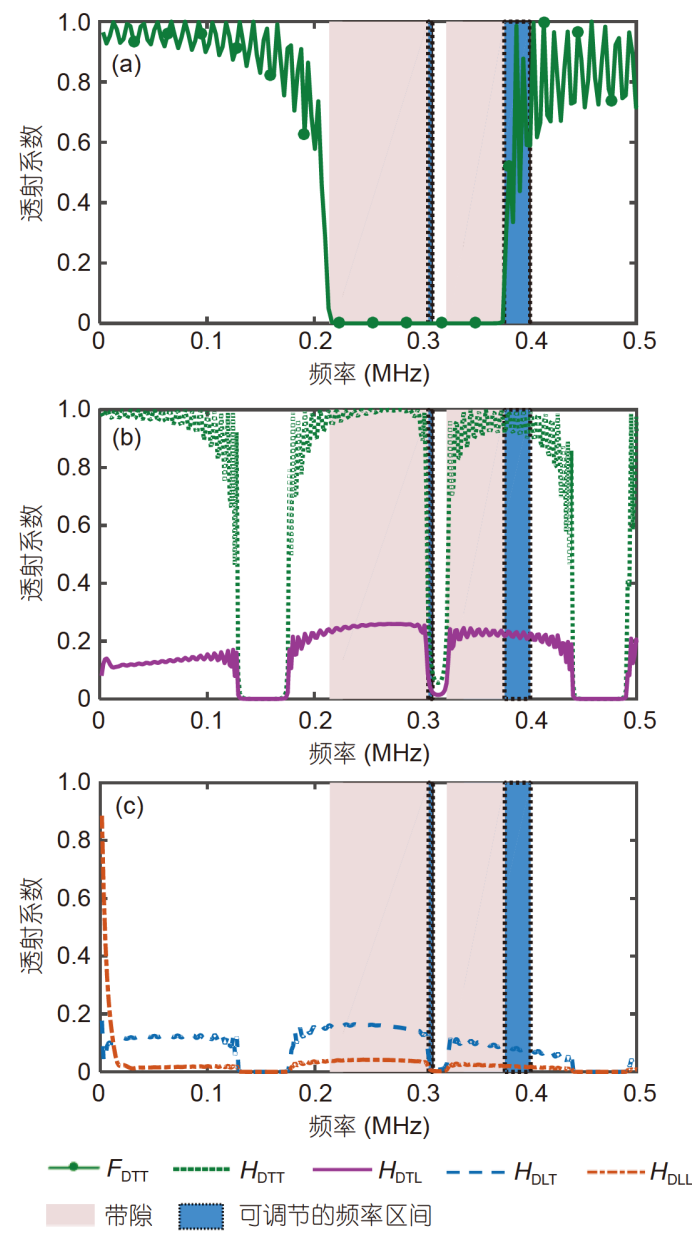

图 5 (网络版彩色) 人射波从右边界人射时, 含非完好界面的非线性 弹性波超材料的非互易传输特性. (a) 基频波; 二次横波(b)和二次纵 波(c)所产生的横波和纵波的透射系数

Figure 5 (Color online) Nonreciprocal transmission in the nonlinear elastic wave metamaterial with imperfect interfaces for the incident wave from the right boundary. (a) The fundamental wave; the transmission coefficients of the generated transverse and longitudinal waves for the incident transverse wave (b) and the incident longitudinal wave (c) with double frequency

\section{参考文献}

1 Zhao J, Pan Y, Zhong Z. Theoretical study of shear horizontal wave propagation in periodically layered piezoelectric structure. J Appl Phys, 2012, 111: 064906

2 Kutsenko A A, Shuvalov A L, Norris A N. Converging bounds for the effective shear speed in 2D phononic crystals. J Elast, 2013, 113: 179-191

3 Witarto W, Nakshatrala K B, Mo Y L. Global sensitivity analysis of frequency band gaps in one-dimensional phononic crystals. Mech Mater, 2019, 134: $38-53$

4 Zhang G Y, Gao X L, Bishop J E, et al. Band gaps for elastic wave propagation in a periodic composite beam structure incorporating microstructure and surface energy effects. Composite Struct, 2018, 189: 263-272

5 Liu M, Zhu W D. Nonlinear transformation-based broadband cloaking for flexural waves in elastic thin plates. J Sound Vib, 2019, 445: 270-287

6 Willis J R. Negative refraction in a laminate. J Mech Phys Solids, 2016, 97: 10-18

7 Daraio C, Nesterenko V F, Herbold E B, et al. Tunability of solitary wave properties in one-dimensional strongly nonlinear phononic crystals. Phys Rev E, 2006, 73: 026610 
8 Iwai A, Nakamura Y, Sakai O. Enhanced generation of a second-harmonic wave in a composite of metamaterial and microwave plasma with various permittivities. Phys Rev E, 2015, 92: 033105

9 Jiao W, Gonella S. Mechanics of inter-modal tunneling in nonlinear waveguides. J Mech Phys Solids, 2018, 111: 1-17

10 Deng M, Xiang Y. Analysis of second-harmonic generation by primary horizontal shear modes in layered planar structures with imperfect interfaces. J Appl Phys, 2013, 113: 043513

11 Liang B, Guo X S, Tu J, et al. An acoustic rectifier. Nat Mater, 2010, 9: 989-992

12 Boechler N, Theocharis G, Daraio C. Bifurcation-based acoustic switching and rectification. Nat Mater, 2011, 10: 665-668

13 Li X F, Ni X, Feng L, et al. Tunable unidirectional sound propagation through a sonic-crystal-based acoustic diode. Phys Rev Lett, 2011, 106: 084301

14 Chen Y, Wu B, Su Y, et al. Tunable two-way unidirectional acoustic diodes: Design and simulation. J Appl Mech, 2019, 86: 031010

15 Li Z N, Wang Y Z, Wang Y S. Nonreciprocal phenomenon in nonlinear elastic wave metamaterials with continuous properties. Int J Solids Struct, 2018, 150: 125-134

16 Deng M X. Nonlinear Lamb waves in layered structures with imperfect interfaces (in Chinese). Acta Acust, 2007, (3): 205-211 [邓明晰. 弱界面分 层结构中的非线性兰姆波. 声学学报, 2007, (3): 205-211]

17 Guo X, Wei P, Li L. Dispersion relations of elastic waves in one-dimensional piezoelectric phononic crystal with mechanically and dielectrically imperfect interfaces. Mech Mater, 2016, 93: 168-183

18 Rokhlin S I, Wang L. Stable recursive algorithm for elastic wave propagation in layered anisotropic media: Stiffness matrix method. J Acoust Soc Am, 2002, 112: 822-834

19 Deng M. Cumulative second-harmonic generation of Lamb-mode propagation in a solid plate. J Appl Phys, 1999, 85: 3051-3058

20 Rose J L. Ultrasonic Waves in Solid Media. Cambridge: Cambridge University Press, 1999 


\title{
Nonreciprocal transmission for the SH wave in a nonlinear elastic wave metamaterial with imperfect interfaces
}

\author{
Zhenni $\mathrm{Li}^{1}$, Yize Wang ${ }^{2 *} \&$ Yuesheng Wang ${ }^{1,2}$ \\ ${ }^{1}$ Institute of Engineering Mechanics, School of Civil Engineering, Beijing Jiaotong University, Beijing 100044, China; \\ ${ }^{2}$ Department of Mechanics, School of Mechanical Engineering, Tianjin University, Tianjin 300350, China \\ * Corresponding author, E-mail: wangyize@tju.edu.cn
}

During the past decades, phononic crystals and elastic wave metamaterials as the artificial periodic structures have received considerable attention. Due to their distinguishing properties, these structures can be used for the wave energy manipulation, e.g., band gaps, elastic wave cloaking and topological state, etc. A well-known characteristic is the band gap which means that the elastic wave cannot propagate in some certain frequency regions. Based on this property, many engineering applications can be achieved, e.g., filtering and wave isolation, etc. The investigations mentioned above are mainly focused on the linear elastic problems which cannot present the nonlinear wave properties. However, the nonlinearity appearing in the materials and structures can bring new and interesting wave phenomena. One of the nonlinear characteristics is the generation of the higher order harmonics. It can be applied to the nonlinear nondestructive testing. Recently, increasing attention has been focused on the nonlinear wave motion, which can result in the nonlinear band gap and acoustic diode.

The new concept named as the acoustic diode has been presented by the combination of the band gap and material nonlinearity, which permits the wave propagation in only one direction. It can be achieved when the fundamental wave sits in the stop band but the second harmonic belongs to the pass band. However, the acoustic wave has one direction displacement component and usually propagates in the air or liquid. Thus we wonder whether the nonreciprocal phenomenon of the elastic wave can be achieved in the solid materials. The problem will become more interesting and necessary because of the coupling displacements with the vector characteristic in solids. In our previous work, we have discussed the nonreciprocal transmission for the incident SH and in-plane elastic waves in a nonlinear elastic wave metamaterial. The interface in the consecutive layers is always assumed as perfect, which means that the displacement and stress components are continuous across the interface. But perfect interfaces may become imperfect in practice during the manufacture, which indicates that the interfacial property on the band gaps is obvious. Then the imperfect interface can offer a new opportunity to tune the nonreciprocal transmission of the elastic wave which depends on the band gap property.

In this work, the nonreciprocal transmission for the SH wave in a layered nonlinear elastic wave metamaterial with the imperfect interfaces is investigated. The combination of the structural asymmetry and material nonlinearity breaks the inherent reciprocity of the classic wave system. The second harmonic can be generated by the interaction between the incident SH wave and material nonlinearity. Based on the Bloch's law and stiffness matrix method, the band gaps and transmission coefficients of both the fundamental and second harmonic waves are obtained. The effects of the interfacial properties on the nonlinear phononic crystal and elastic wave metamaterial are discussed. We find that comparing with the perfect interface system, the central frequency of the nonreciprocal regions shifts towards the low frequency region by the effects of imperfect interfaces. This present work is expected to be helpful to design the practical devices with the tuning nonreciprocal transmission of the elastic wave.

nonlinear elastic wave metamaterial, nonreciprocal transmission, second harmonic, imperfect interface, band gap doi: 10.1360/TB-2019-0483 